\title{
RELATIVELY COMPACT-LIKE PERTURBATIONS, ESSENTIAL SPECTRA AND APPLICATION
}

\author{
KHALID LATRACH and J. MARTIN PAOLI
}

(Received 20 May 2002; revised 16 April 2003)

\author{
Communicated by G. Willis
}

\begin{abstract}
The purpose of this paper is to provide a detailed treatment of the behaviour of essential spectra of closed densely defined linear operators subjected to additive perturbations not necessarily belonging to any ideal of the algebra of bounded linear operators. If $A$ denotes a closed densely defined linear operator on a Banach space $X$, our approach consists principally in considering the class of $A$-closable operators which, regarded as operators in $\mathscr{L}\left(X_{A}, X\right)$ (where $X_{A}$ denotes the domain of $A$ equipped with the graph norm), are contained in the set of $A$-Fredholm perturbations (see Definition 1.2). Our results are used to describe the essential spectra of singular neutron transport equations in bounded geometries.
\end{abstract}

2000 Mathematics subject classification: primary 47A53, 47A55, 47G20.

\section{Introduction and preliminaries}

Let $X$ and $Y$ be two Banach spaces. By an operator $A$ from $X$ into $Y$ we mean a linear operator with domain $D(A) \subseteq X$ and range contained in $Y$. We denote by $\mathscr{C}(X, Y)$ (respectively $\mathscr{L}(X, Y)$ ) the set of all closed, densely defined (respectively bounded) linear operators from $X$ into $Y$. The subset of all compact (respectively weakly compact) operators of $\mathscr{L}(X, Y)$ is designated by $\mathscr{K}(X, Y)$ (respectively $\mathscr{W}(X, Y)$ ). If $A \in \mathscr{C}(X, Y)$, we write $N(A) \subseteq X$ and $R(A) \subseteq Y$ for the null space and range of $A$. We set $\alpha:=\operatorname{dim} N(A), \beta:=\operatorname{codim} R(A)$. Let $A \in \mathscr{C}(X, Y)$ with a closed range. Then $A$ is a $\Phi_{+}$-operator $\left(A \in \Phi_{+}(X, Y)\right)$ if $\alpha(A)<\infty$, and $A$ is a $\Phi_{-}$operator $\left(A \in \Phi_{-}(X, Y)\right)$ if $\beta(A)<\infty ; \Phi(X, Y)=\Phi_{+}(X, Y) \cap \Phi_{-}(X, Y)$ is the class of Fredholm operators while $\Phi_{ \pm}(X, Y)$ denotes the set $\Phi_{+}(X, Y) \cup \Phi_{-}(X, Y)$. For $A \in \Phi(X, Y)$, the index of $A$ is defined by $i(A)=\alpha(A)-\beta(A)$. If $X=Y$, then

(c) 2004 Australian Mathematical Society $1446-7887 / 04 \$ A 2.00+0.00$ 
$\mathscr{L}(X, Y), \mathscr{K}(X, Y), \mathscr{W}(X, Y), \mathscr{C}(X, Y), \Phi_{+}(X, Y), \Phi_{ \pm}(X, Y)$, and $\Phi(X, Y)$ are replaced, respectively, by $\mathscr{L}(X), \mathscr{K}(X), \mathscr{W}(X), \mathscr{C}(X), \Phi_{+}(X), \Phi_{ \pm}(X)$ and $\Phi(X)$. Let $A \in \mathscr{C}(X)$. The spectrum of $A$ will be denoted by $\sigma(A)$. The resolvent set of $A$, $\rho(A)$, is the complement of $\sigma(A)$ in the complex plane. A complex number $\lambda$ is in $\Phi_{+A}, \Phi_{-A}, \Phi_{ \pm A}$ or $\Phi_{A}$ if $\lambda-A$ is in $\Phi_{+}(X), \Phi_{-}(X), \Phi_{ \pm}(X)$ or $\Phi(X)$ respectively. For the properties of these sets we refer to [7, 12] or [24].

For self-adjoint operators in a Hilbert space, there seems to be only one reasonable way of defining the essential spectrum: the set of all points of the spectrum that are not isolated eigenvalues of finite algebraic multiplicity (see, for example, [22, 30]). If $X$ is a Banach space and $A \in \mathscr{C}(X)$, various notions of essential spectrum appear in the literature, most are enlargement of the continuous spectrum. Define the sets

$$
\begin{aligned}
& \sigma_{e 1}(A):=\left\{\lambda \in \mathbb{C} \text { such that } \lambda-A \notin \Phi_{+}(X)\right\}:=\mathbb{C} \backslash \Phi_{+A}, \\
& \sigma_{e 2}(A):=\left\{\lambda \in \mathbb{C} \text { such that } \lambda-A \notin \Phi_{-}(X)\right\}:=\mathbb{C} \backslash \Phi_{-A}, \\
& \sigma_{e 3}(A):=\left\{\lambda \in \mathbb{C} \text { such that } \lambda-A \notin \Phi_{ \pm}(X)\right\}:=\mathbb{C} \backslash \Phi_{ \pm A}, \\
& \sigma_{e 4}(A):=\{\lambda \in \mathbb{C} \text { such that } \lambda-A \notin \Phi(X)\}:=\mathbb{C} \backslash \Phi_{A}, \\
& \sigma_{e 5}(A):=\mathbb{C} \backslash \rho_{5}(A), \\
& \sigma_{e 6}(A):=\mathbb{C} \backslash \rho_{6}(A),
\end{aligned}
$$

where $\rho_{5}(A):=\left\{\lambda \in \Phi_{A} ; i(\lambda-A)=0\right\}$ and $\rho_{6}(A):=\left\{\lambda \in \rho_{5}(A)\right.$ such that all scalars near $\lambda$ are in $\rho(A)\}$. We call $\sigma_{e 1}(\cdot)$ and $\sigma_{e 2}(\cdot)$ the Gustafson and Weidman essential spectra [10] and $\sigma_{e 3}(\cdot)$ the Kato essential spectrum [13]. Further, $\sigma_{e 4}(\cdot)$ is the Wolf essential spectrum [10,23, 30], $\sigma_{e 5}(\cdot)$ is the Schechter essential spectrum $[10,23,24]$, and $\sigma_{e 6}(\cdot)$ is the Browder essential spectrum $[10,23]$. Note that all these sets are closed and, in general, we have

$$
\sigma_{e 1}(A) \cap \sigma_{e 2}(A)=\sigma_{e 3}(A) \subseteq \sigma_{e 4}(A) \subseteq \sigma_{e 5}(A) \subseteq \sigma_{e 6}(A) \subseteq \sigma(A) .
$$

But if $X$ is a Hilbert space and $A$ is self-adjoint, then all these sets coincide.

An operator $T \in \mathscr{L}(X, Y)$ is said to be strictly singular if, for every infinite dimensional subspace $M$ of $X$, the restriction of $T$ to $M$ is not a homeomorphism. Let $\mathscr{S}(X, Y)$ denote the set of all strictly singular operators from $X$ into $Y$. For a detailed study of the properties of strictly singular operators we refer to [12]. Note that $\mathscr{S}(X, Y)$ is a closed subspace of $\mathscr{L}(X, Y)$. In general, strictly singular operators are not compact (see [8]) and, if $X=Y, \mathscr{S}(X)$ is a closed two-sided ideal of $\mathscr{L}(X)$ containing $\mathscr{K}(X)$.

If $N$ is a closed subspace of a Banach space $Z$, we denote by $\pi_{N}^{Z}$ the quotient map $Z \rightarrow Z / N$. The codimension of $N, \operatorname{codim}(N)$, is defined to be the dimension of the vector space $Z / N$.

An operator $T \in \mathscr{L}(X, Y)$ is said to be strictly cosingular if there exists no closed subspace $N$ of $Y$ with $\operatorname{codim}(N)=\infty$ such that $\pi_{N}^{Y} T: X \rightarrow Y / N$ is surjective. Let 
$C \mathscr{S}(X, Y)$ denote the set of strictly cosingular operators from $X$ into $Y$. This class of operators was introduced by Pelczynski [21]. It forms a closed subspace of $\mathscr{L}(X, Y)$ and is a closed two-sided ideal of $\mathscr{L}(X)$ if $X=Y$ (see [27]).

Let $\Phi^{b}(X, Y), \Phi_{+}^{b}(X, Y)$ and $\Phi_{-}^{b}(X, Y)$ designate the sets $\Phi(X, Y) \cap \mathscr{L}(X, Y)$, $\Phi_{+}(X, Y) \cap \mathscr{L}(X, Y)$ and $\Phi_{-}(X, Y) \cap \mathscr{L}(X, Y)$, respectively.

DEFINITION 1.1. Let $F \in \mathscr{L}(X, Y) . \quad F$ is called a Fredholm perturbation if $U+F \in \Phi^{b}(X, Y)$ whenever $U \in \Phi^{b}(X, Y) . F$ is called an upper (respectively lower) Fredholm perturbation if $F+U \in \Phi_{+}^{b}(X, Y)$ (respectively $\Phi_{-}^{b}(X, Y)$ ) whenever $U \in \Phi_{+}^{b}(X, Y)$ (respectively $\Phi_{-}^{b}(X, Y)$ ).

The sets of Fredholm, upper semi-Fredholm and lower semi-Fredholm perturbations are denoted by $\mathscr{F}^{b}(X, Y), \mathscr{F}_{+}^{b}(X, Y)$ and $\mathscr{F}_{-}^{b}(X, Y)$, respectively. These classes of operators were introduced and investigated in [8]. In particular, it is shown that $\mathscr{F}_{+}^{b}(X, Y)$ and $\mathscr{F}^{b}(X, Y)$ are closed subsets of $\mathscr{L}(X, Y)$, and if $X=Y$, then $\mathscr{F}_{+}^{b}(X)$ and $\mathscr{F}^{b}(X)$ are closed two-sided ideals of $\mathscr{L}(X)$. We recall the following useful result due to Gohberg, Markus and Fel'dman [8, pages 69-70].

Proposition 1.1. Let $X, Y$ and $Z$ be three Banach spaces. If at least one of the sets $\Phi^{b}(X, Y)$ and $\Phi^{b}(Y, Z)$ is not empty, then

$$
F_{1} \in \mathscr{F}^{b}(X, Y), \quad A \in \mathscr{L}(Y, Z) \quad\left(F_{2} \in \mathscr{F}^{b}(Y, Z), \quad B \in \mathscr{L}(X, Y)\right)
$$

imply

$$
A F_{1} \in \mathscr{F}^{b}(X, Z) \quad\left(F_{2} B \in \mathscr{F}^{b}(X, Z)\right) .
$$

For $A \in \mathscr{C}(X)$, the graph norm of $A$ is defined by

$$
\|x\|_{A}=\|x\|+\|A x\|, \quad x \in \mathscr{D}(A),
$$

where $\mathscr{D}(A)$ denotes the domain of $A$. It follows from the closedness of $A$ that $\mathscr{D}(A)$ endowed with the norm $\|\cdot\|_{A}$ is a Banach space. In this new space, denoted by $X_{A}$, the operator $A$ satisfies $\|A x\| \leq\|x\|_{A}$, and consequently, $A \in \mathscr{L}\left(X_{A}, X\right)$. Let $J$ be a linear operator on $X$. If $\mathscr{D}(A) \subset \mathscr{D}(J)$, then $J$ will be called $A$-defined. If $J$ is $A$-defined, we will denote by $\hat{J}$ its restriction to $\mathscr{D}(A)$. Moreover, if $\hat{J} \in \mathscr{L}\left(X_{A}, X\right)$, we say that $J$ is $A$-bounded. One checks easily that if $J$ is closed (or closable) (see [13, Remark 1.5, page 191]), then $J$ is $A$-bounded.

REMARK 1.1. We say that an operator $J$ is $A$-closed if $x_{n} \rightarrow x, A x_{n} \rightarrow y, J x_{n} \rightarrow z$ for $\left\{x_{n}\right\} \subseteq \mathscr{D}(A)$ implies that $x \in \mathscr{D}(J)$ and $J x=z$. It will be called $A$-closable if $x_{n} \rightarrow 0, A x_{n} \rightarrow 0, J x_{n} \rightarrow z$ implies $z=0$. It is evident that if $J$ is closed (respectively closable), then $J$ is $A$-closed (respectively $A$-closable). Note, however, that if $A$ is closed, by [23, Lemma 2.1], we get the equivalence between the following three concepts: (i) $J$ is $A$-closed, (ii) $J$ is $A$-closable and (iii) $J$ is $A$-bounded. 
DEFINITION 1.2. Let $X$ be a Banach space, $A \in \mathscr{C}(X)$ and let $F$ be an $A$ defined linear operator on $X$. We say that $F$ is an A-Fredholm perturbation if $\hat{F} \in \mathscr{F}^{b}\left(X_{A}, X\right) . F$ is called an upper (respectively lower) $A$-semi-Fredholm perturbation if $\hat{F} \in \mathscr{F}_{+}^{b}\left(X_{A}, X\right)$ (respectively $\hat{F} \in \mathscr{F}_{-}^{b}\left(X_{A}, X\right)$ ).

Let $A \mathscr{F}(X), A \mathscr{F}_{+}(X)$ and $A \mathscr{F}_{-}(X)$ designate the sets of $A$-Fredholm, upper $A$-semi-Fredholm and lower $A$-semi-Fredholm perturbations, respectively.

Definition 1.3. Let $A \in \mathscr{C}(X)$ and let $J$ be an arbitrary $A$-defined linear operator on $X$. We say that $J$ is $A$-compact (respectively $A$-weakly compact, $A$-strictly singular, A-strictly cosingular) if $\hat{J} \in \mathscr{K}\left(X_{A}, X\right)$ (respectively $\hat{J} \in \mathscr{W}\left(X_{A}, X\right)$, $\left.\hat{J} \in \mathscr{S}\left(X_{A}, X\right), \hat{J} \in C \mathscr{S}\left(X_{A}, X\right)\right)$.

Let $A \mathscr{K}(X), A \mathscr{W}(X), A \mathscr{S}(X)$ and $A C \mathscr{S}(X)$ denote, respectively, the sets of $A$ compact, $A$-weakly compact, $A$-strictly singular and $A$-strictly cosingular operators on $X$.

REMARK 1.2. Clearly, if $J$ is bounded, then $J$ is $A$-bounded, $J$ is compact (respectively weakly compact, strictly singular, strictly cosingular) implies that $J$ is $A$-compact (respectively $A$-weakly compact, $A$-strictly singular, $A$-strictly cosingular).

REMARK 1.3. Notice that the concept of $A$-boundedness, $A$-compactness, $A$-strict singularity, $A$-strict cosingularity and $A$-Fredholmness are not connected with the operator $A$ itself, but only with its domain. Note also that an easy consequence of Definition 1.2 and the inclusions in [8, page 69] that

$$
\begin{aligned}
& A \mathscr{K}(X) \subseteq A \mathscr{S}(X) \subseteq A \mathscr{F}_{+}(X) \subseteq A \mathscr{F}(X), \\
& A \mathscr{K}(X) \subseteq A C \mathscr{S}(X) \subseteq A \mathscr{F}_{-}(X) \subseteq A \mathscr{F}(X)
\end{aligned}
$$

The inclusion $A \mathscr{S}(X) \subseteq A \mathscr{F}_{+}(X)$ (respectively $A C \mathscr{S}(X) \subseteq A \mathscr{F}_{-}(X)$ ) was established in [12] (respectively [27]).

When dealing with essential spectra of closed densely defined linear operators on Banach spaces, one of the main problems consists in studying the invariance of the essential spectra of these operators subjected to various kinds of perturbations. Among the works in this direction we quote, for example, $[10,15,16,17,22,24,30]$ (see also the references therein). This work is a continuation of [17], where we can find a detailed treatment of the behaviour of essential spectra of such operators subjected to additive perturbations belonging to arbitrary closed two-sided ideals of $\mathscr{L}(X)$ contained in the set of Riesz operators (see [17, page 281]). It is inspired by the work published in [15] and [16], where $A$-weakly compact and $A$-strictly singular 
perturbations ( $A$ denotes a closed densely defined linear operator) were considered on Banach spaces which possess the Dunford-Pettis property, and on $L_{p}$ spaces, respectively. Our main objective here is to extend the results obtained in [15, Section 4] and [16, Section 2] to arbitrary Banach spaces and to fit them into a more general framework. The extension consists principally in the possibility of considering the class of $A$-bounded operators which, regarded as operators in $\mathscr{L}\left(X_{A}, X\right)$, are contained in one of the sets $A \mathscr{F}_{+}(X), A \mathscr{F}_{-}(X), A \mathscr{F}_{+}(X) \cap A \mathscr{F}_{-}(X)$ or $A \mathscr{F}(X)$. Accordingly, using the same strategy as in [17], we find conditions which generalize previous ones discussed in $[15,16]$. In contrast to the proofs of the results obtained in [15] and [16], which use the geometric properties of Banach spaces considered, our analysis applies to all Banach spaces regardless of their specific properties and to a wide family of operators including, in particular, the sets $A \mathscr{K}(X), A \mathscr{W}(X), A \mathscr{S}(X)$ and $A C \mathscr{S}(X)$. Note also that our results provide a natural extension to those obtained in [17].

In the last section we consider the following singular neutron transport operator

$$
\begin{aligned}
A \psi(x, \xi) & =-v \frac{\partial \psi}{\partial x}(x, v)-\sigma(v) \psi(x, v)+\int_{\mathbb{R}^{n}} \kappa\left(v, v^{\prime}\right) \psi\left(x, v^{\prime}\right) d v^{\prime} \\
& =T \psi(x, v)+K \psi(x, v),
\end{aligned}
$$

where $(x, v) \in D \times \mathbb{R}^{n}$. Here $D$ is an open bounded subset of $\mathbb{R}^{n}$ and $d \mu(\cdot)$ is a positive Radon measure on $\mathbb{R}^{N}$. This operator describes the transport of particles (neutrons, photons, molecules of gas, etc.) in the domain $D$. The function $\psi(x, v)$ represents the number (or probability) density of gas particles having the position $x$ and the velocity $v$. The functions $\sigma(\cdot)$ and $\kappa(\cdot, \cdot)$ are called, respectively, the collision frequency and the scattering kernel. The operator $A$ is supplemented with vacuum boundary conditions that is $\psi_{\Gamma_{-}}=0$ with $\Gamma_{-}=\left\{(x, v) \in \partial D \times \mathbb{R}^{n}, v \cdot v_{x}<0\right\}$, where $v_{x}$ stands for the outer unit normal vector at $x \in \partial D$.

Here the functions $\sigma(\cdot)$ and $\kappa(\cdot, \cdot)$ will be assumed to be unbounded. More precisely, we will assume that there exist a closed subset $\mathscr{O} \subseteq \mathbb{R}^{n}$ with zero $d \mu$ measure and a constant $\sigma_{0}>0$ such that

$$
\begin{gathered}
\sigma(\cdot) \in L_{\mathrm{loc}}^{\infty}\left(\mathbb{R}^{n} \backslash \mathscr{O}\right), \quad \sigma(v)>\sigma_{0} \text { a.e. } \\
{\left[\int_{\mathbb{R}^{n}}\left(\frac{\kappa\left(\cdot, v^{\prime}\right)}{\sigma\left(v^{\prime}\right)^{1 / p}}\right)^{q} d \mu\left(v^{\prime}\right)\right]^{1 / q} \in L_{p}\left(\mathbb{R}^{n}\right),}
\end{gathered}
$$

where $q$ denotes the conjugate exponent of $p$. These assumptions were motivated by free gas models (see $[4,25]$ ) and were already used by Chabi and MokhtarKharroubi [2] in $L_{1}$ spaces and by Lods [18] in the case of $L_{p}$ spaces (see also [19, Chapter 9] or [28]). The first part of the condition (1.2) means that the singularities of the collision frequency are contained in a set of zero $d \mu$ measure. In fact, unbounded and nonnegative collision frequencies act as strong absorbers which allow the unboundedness of the collision operator. We also deal with abstract velocity measures 
$d \mu(\cdot)$, hence our analysis works for continuous models (Lebesgue measure on open subsets of $\mathbb{R}^{n}$ ), multigroup models (surface Lebesgue measures on spheres) as well as discrete ones (finite sum of Dirac measures).

In $[2,18,19]$ the authors discussed essentially the asymptotic spectrum of $A$. The main goal of this paper is to apply the results of Section 2 to describe the essential spectra of the operator $A$ subjected to assumptions (1.2) and (1.3). The main result of Section 4 is Theorem 4.1. It asserts that if conditions (1.2) and (1.3) are satisfied, the hyperplanes of $\mathbb{R}^{n}$ have zero $d \mu$-measure (that is, for each $e \in S^{n-1}$, $d \mu\left\{v \in \mathbb{R}^{n} ;\right.$ v.e $\left.=0\right\}=0$, where $S^{n-1}$ denotes the unit sphere of $\left.\mathbb{R}^{n}\right)$; and if further the collision operator $K$ is compact from $L_{p}\left(\mathbb{R}^{n}, \sigma(v) d \mu(v)\right)$ into $L_{p}\left(\mathbb{R}^{n}, d \mu(v)\right)$, then $\sigma_{e i}(A)=\left\{\lambda \in \mathbb{C}\right.$ such that $\left.\operatorname{Re} \lambda \leq-\sigma_{0}\right\}$ for $i=1, \ldots, 5$. Our analysis is based essentially on Proposition 2.1, Proposition 4.1 and the knowledge of the essential spectra of the streaming operator $T$.

\section{Main results}

Let $X$ be a Banach space and $A \in \mathscr{C}(X)$. In what follows we shall be concerned with $A$-bounded perturbations belonging to $A \mathscr{F}(X)$. It is a wide class of operators which contains all the classical perturbation classes considered in the literature (see Remarks 1.2-1.3). It is worth remarking that, according to Remark 1.1, operators in $A \mathscr{F}(X)$ need not be closed.

We are now ready to state the main result of this paper which generalizes [17, Theorem 3.1].

THEOREM 2.1. Let $A \in \mathscr{C}(X)$ and let $J$ be an operator on $X$. The following statements are satisfied.

(i) If $J \in A \mathscr{F}(X)$, then $\sigma_{e i}(A)=\sigma_{e i}(A+J), i=4,5$. Moreover, if $C \sigma_{e s}(A)$ (the complement of $\sigma_{e s}(A)$ ) is connected and neither $\rho(A)$ nor $\rho(A+J)$ is empty, then $\sigma_{e 6}(A)=\sigma_{e 6}(A+J)$. Further,

(ii) if $J \in A \mathscr{F}_{+}(X)$, then $\sigma_{e 1}(A)=\sigma_{e 1}(A+J)$;

(iii) if $J \in A \mathscr{F}_{-}(X)$ or $J^{*} \in A \mathscr{F}_{+}\left(X^{*}\right)$, then $\sigma_{e 2}(A)=\sigma_{e 2}(A+J)$;

(iv) if $J \in A \mathscr{F}_{+}(X) \bigcap A \mathscr{F}_{-}(X)$, then $\sigma_{e 3}(A)=\sigma_{e 3}(A+J)$.

Let $X$ be a Banach space. We say that $X$ possesses the Dunford-Pettis property (for short, property DP) if, for each Banach space $Y$, every weakly compact operator $T: X \rightarrow Y$ takes weakly compact sets in $X$ into norm compact sets of $Y$. It is well known that any $L_{1}$ space has the property DP. Also, if $\Omega$ is a compact Hausdorff space then $C(\Omega)$ has the property DP. For further examples we refer to [5] or [6, pages 494, 497, 508 and 511$]$. 
REMARK 2.1. Item (i) was first proved in the particular case when $J \in A \mathscr{K}(X)$ in [23, Theorems 2.1 and 2.6]. This is due to the fact that $A \mathscr{K}(X) \subseteq A \mathscr{F}_{+}(X) \cap$ $A \mathscr{F}_{-}(X)$. If $X$ is an $L_{p}$ space, $1 \leq p \leq \infty$, and $A \in \mathscr{C}(X)$, then assertions (i) and (ii) are valid for all $J \in A \mathscr{S}(X)$ [16, Theorem 2.1]. The same is true for the spaces $l_{p}, 1 \leq p<\infty, c_{0}$ and $C(\Omega)$, where $\Omega$ is a compact Hausdorff space. Also, using $A$-weakly compact perturbations, statements (i) and (iii) were established in [15, Theorem 4.1] for Banach spaces which possess the property DP.

Notice that in most applications (transport operators, Schrödinger operators, operators arising in dynamic populations etc. see $[3,9,17,22,24])$, we deal with operators $A$ and $B$ such that $B=A+J$, where $A \in \mathscr{C}(X)$ (often is the generator of a strongly continuous semigroup) and $J$ is, in general, a closed (or closable) $A$-defined operator not necessarily bounded. The next proposition provides a practical criterion for the invariance of essential spectra for such operators, which is useful in applications (see Section 4).

Proposition 2.1. Let $A \in \mathscr{C}(X)$ and let $J$ be an $A$-bounded operator on $X$. Let $\mathscr{I}(X)$ be any nonzero closed two-sided ideal of $\mathscr{L}(X)$ satisfying $\mathscr{I}(X) \subseteq \mathscr{F}(X)$, where $\mathscr{F}(X)$ denotes the ideal of Fredholm perturbations, and assume that there is a complex number $\lambda \in \rho(A)$ such that $r_{\sigma}\left(J(\lambda-A)^{-1}\right)<1$.

(i) If $J(\lambda-A)^{-1} \in \mathscr{I}(X)$, then $\sigma_{e i}(A+J)=\sigma_{e i}(A), i=4,5$.

Moreover,

(ii) if $\mathscr{I}(X) \subseteq \mathscr{F}_{+}(X)$, then $\sigma_{e 1}(A+J)=\sigma_{e 1}(A)$;

(iii) if $\mathscr{I}(X) \subseteq \mathscr{F}_{-}(X)$, then $\sigma_{e 2}(A)=\sigma_{e 2}(A+J)$;

(iv) if $\mathscr{I}(X) \subseteq \mathscr{F}_{+}(X) \bigcap \mathscr{F}_{-}(X)$, then $\sigma_{e 3}(A)=\sigma_{e 3}(A+J)$.

Proof. Let $\lambda \in \rho(A)$. Since $J$ is $A$-bounded, according to [23, Lemma 2.1], $J(\lambda-A)^{-1}$ is a closed linear operator defined on all $X$ and therefore bounded by the closed graph theorem. On the other hand, the assumption $r_{\sigma}\left(J(\lambda-A)^{-1}\right)<1$ implies that $\lambda \in \rho(A+J)$ and

$$
(\lambda-A-J)^{-1}-(\lambda-A)^{-1}=\sum_{n \geq 1}(\lambda-A)^{-1}\left[J(\lambda-A)^{-1}\right]^{n} .
$$

Clearly, if $J(\lambda-A)^{-1} \in \mathscr{I}(X)$, then the closedness of $\mathscr{I}(X)$ implies that $(\lambda-A-J)^{-1}-(\lambda-A)^{-1} \in \mathscr{I}(X)$. Now items (i)-(iv) follow immediately from [17, Theorem 3.2].

Let us now recall another definition of the Schechter essential spectrum (see, for example, [23, 24]). It asserts that if $A \in \mathscr{C}(X)$, then $\sigma_{e 5}(A)$ is the largest subset 
of $\sigma(A)$ which remains invariant under compact perturbations, that is,

$$
\sigma_{e 5}(A)=\bigcap_{K \in \mathscr{X}(X)} \sigma(A+K) .
$$

The equivalence between (2.1) and the definition of $\sigma_{e 5}(\cdot)$ given in the Introduction was established in [24, Theorem 5.4, page 180]. In [17, Theorem 3.4], (2.1) is somewhat relaxed. Actually, it is proved that

$$
\sigma_{e s}(A)=\bigcap_{J \in \mathscr{J}(X)} \sigma(A+J),
$$

where $\mathscr{J}(X)$ is a two sided ideal of $\mathscr{L}(X)$ satisfying $\mathscr{K}(X) \subseteq \mathscr{J}(X) \subseteq \mathscr{F}(X)$. Nevertheless, a careful examination of the proof of Theorem 5.4 in [24, page 180] shows that (2.1) remains valid if we replace $\mathscr{K}(X)$ by $\mathscr{F}_{0}(X)$ (the ideal of finite rank operators). So, $\mathscr{F}_{0}(X)$ is the minimal subset of $\mathscr{L}(X)$ (in the sense of inclusion) for which (2.2) holds true.

In the next theorem we will give a sharper form of (2.2) which extends it to $A$-bounded perturbations contained in $A \mathscr{F}(X)$. To do so, we will assume that

$$
\mathscr{F}_{0}(X) \subseteq \mathscr{J}(X) \subseteq A \mathscr{F}(X) .
$$

THEOREM 2.2. Let $A \in \mathscr{C}(X)$ and let $\mathscr{J}(X)$ be any subset of operators satisfying (2.3). Then $\sigma_{e s}(A)=\bigcap_{J \in \mathscr{I}(X)} \sigma(A+J)$.

REMARK 2.2. This theorem may be viewed as an extension of [15, Theorem 4.2] and [16, Theorem 2.2]. In fact, in [16] (respectively [15]) it is proved that in the case when $X$ is an $L_{p}$ space (respectively has the property DP), the definition of $\sigma_{e s}(\cdot)$ can be stated in terms of $A$-strictly singular (respectively $A$-weakly compact) perturbations. Since $A \mathscr{S}\left(L_{p}\right) \subseteq A \mathscr{F}\left(L_{p}\right)(1 \leq p \leq \infty)$ and $A \mathscr{W}(X) \subseteq A \mathscr{F}(X)$, if $X$ has the property DP, then these two results are particular cases of our theorem. Their proofs depend in a crucial way on the properties and the structure of both the families of operators and the classes of spaces considered, and are different from the proof of the Theorem 2.2 given below.

Finally, we have the following analogue of Proposition 3.2 in [17] which extends it to the case of $A$-Fredholm perturbations.

Proposition 2.2. Let $A \in \mathscr{C}(X)$. If $\sigma_{e 6}(A)=\sigma_{t 5}(A)$, then, for each $J \in A \mathscr{F}(X)$, there is at most a countable set $\mathscr{S}$ of complex numbers such that

$$
\sigma_{e 6}(A+\zeta J)=\sigma_{e 6}(A)
$$

for $\zeta \notin \mathscr{S}$. If $C \sigma_{e 6}(A)$ consists of a finite number of components, then $\mathscr{S}$ is discrete. 
ProOF. It is verbatim the proof of [17, Proposition 3.2]. It suffices only to replace in the text Proposition 3.1 by Lemma 3.1 below.

Remarks 2.3. (1) Let $(\Omega, \Sigma, \mu)$ be a positive measure space and let $X_{p}$ denote the spaces $L_{p}(\Omega, d \mu)$ with $1 \leq p<\infty$. Recall that, if $X=X_{p}$ or $X=C(\Xi)$ (the Banach space of continuous scalar-valued functions on $\Xi$ with the supremum norm where $\Xi$ is a compact Hausdorff space), then $\mathscr{S}(X)=C \mathscr{S}(X)=\mathscr{F}(X)$ (see [16, (2.9) and (2.10)]). So, for these spaces we have $A \mathscr{S}(X)=A C \mathscr{S}(X)=A \mathscr{F}(X)$.

(2) Recall that, following Calkin [1], if $X$ is a separable Hilbert space, then $\mathscr{K}(X)$ is the unique proper nonzero closed two-sided ideal of $\mathscr{L}(X)$. This result also holds true for the spaces $l_{p}, 1 \leq p<\infty$ and $c_{0}$ [8]. Hence if $X$ is one of these spaces, then $\mathscr{K}(X)=\mathscr{F}(X)$, and therefore $A \mathscr{K}(X)$ is largest class of operators which permits to derive the results stated above.

(3) A Banach space $X$ is an h-space if each closed infinite dimensional subspace of $X$ contains a complemented subspace isomorphic to $X$. Any Banach space isomorphic to an h-space is an h-space; $c, c_{0}$ and $l_{p}(1 \leq p<\infty)$ are h-spaces. Let $X$ be an h-space, according to [29, Theorem 6.2], $\mathscr{S}(X)$ is the greatest proper ideal of $\mathscr{L}(X)$. Hence, since $\mathscr{S}(X) \subseteq \mathscr{F}(X)$ we conclude that $\mathscr{S}(X)=\mathscr{F}(X)$. Next, let $A \in \mathscr{C}(X)$, then it follows from Definition 1.3 and Remark 1.3 that $A \mathscr{S}(X)=A \mathscr{F}(X)$. Accordingly, for h-spaces, $A \mathscr{S}(X)$ is the largest family of operators for which the results of this section are valid.

\section{Proofs}

To establish the results stated above we will make use of the following perturbation lemma which is fundamental to our purpose. It generalizes many known perturbation results in the literature.

Lemma 3.1. Let $A \in \mathscr{C}(X)$ and let $J$ be an operator on $X$. Assume that $J \in A \mathscr{F}(X)$.

(i) If $A \in \Phi(X)$, then $A+J \in \Phi(X)$ and $i(A+J)=i(A)$. Moreover,

(ii) if $A \in \Phi_{+}(X)$ and $J \in A \mathscr{F}_{+}(X)$, then $A+J \in \Phi_{+}(X)$;

(iii) if $A \in \Phi_{-}(X)$ and $J \in A \mathscr{F}_{-}(X)$ or $J^{*} \in A \mathscr{F}_{+}\left(X^{*}\right)$, then $A+J \in \Phi_{-}(X)$;

(iv) if $A \in \Phi_{ \pm}(X)$ and $J \in A \mathscr{F}_{+}(X) \cap A \mathscr{F}_{-}(X)$, then $A+J \in \Phi_{ \pm}(X)$.

REMARK 3.1. During the last decades, perturbation theory has experienced great developments motivated by concrete problems arising in different branches of physics and biology see, for example, the works $[7,8,10,12,13,20,22,23,24,27,30]$. 
When dealing with $A$-bounded perturbations, where $A \in \mathscr{C}(X)$, many results in the spirit of the assertions of Lemma 3.1 concerning special classes of operators and particular Banach spaces may be encountered in the literature. In fact, the first result in the spirit of Lemma 3.1 (i) for A-compact operators was established by Nagy [20]. Using Nagy's idea, Gohberg and Krein have obtained items (ii) and (iii) (and consequently (iv)) stated above for the same class of operators [8]. In [12] Kato proved assertion (ii) for $A$-strictly singular operators. If $X$ has the property DP and $J \in A \mathscr{W}(X)$, then Lemma 3.1 (i) and (iii) hold true (see [15]). Recently, it is proved that if $X$ is an $L_{p}$ space $(1 \leq p \leq \infty)$ and $J \in A \mathscr{S}(X)$, then statements (i) and (ii) are valid (see [16]). Note that in our case, Vladimirskii's result (see [27, Corollary 1]) writes $A C \mathscr{S}(X) \subseteq A \mathscr{F}_{-}(X)$, and therefore (i) and (iii) hold true for $A$-strictly cosingular perturbations.

Proof of LEMMA 3.1. Since $A \in \mathscr{C}(X)$ and $J \in A \mathscr{F}(X)$, hence as mentioned above we can regard $A$ and $J$ as operators from $X_{A}$ into $X$. They will be denoted by $\hat{A}$ and $\hat{J}$ respectively. These belong to $\mathscr{L}\left(X_{A}, X\right)$ and we have

$$
\left\{\begin{aligned}
\alpha(\hat{A}) & =\alpha(A), \quad \beta(\hat{A})=\beta(A), \quad R(\hat{A})=R(A), \\
\alpha(\hat{A}+\hat{J}) & =\alpha(A+J), \\
\beta(\hat{A}+\hat{J}) & =\beta(A+J) \quad \text { and } \quad R(\hat{A}+\hat{J})=R(A+J) .
\end{aligned}\right.
$$

Observe that assertion (ii), the first part of (iii) and (iv) are immediate. To prove the second part of (iii) we proceed as follows. Let $A \in \Phi_{-}(X)$. Applying [13, Theorem 5.13, page 234] we infer that $A^{*} \in \Phi_{+}\left(X^{*}\right)$. Moreover, $J^{*} \in A \mathscr{F}_{+}\left(X^{*}\right)$ implies that $A^{*}+J^{*} \in \Phi_{+}\left(X^{*}\right)$. This together with the fact that $\alpha\left(A^{*}+J^{*}\right)=\beta(A+J)$ (use again [13, Theorem 5.13, page 234]) gives the result.

(i) Assume that $A \in \Phi(X)$. Then using (3.1) we infer that $\hat{A} \in \Phi^{b}\left(X_{A}, X\right)$. Hence, it follows from [24, Theorem 1.4, page 108] that there exist $A_{0} \in \mathscr{L}\left(X, X_{A}\right)$ and $K \in \mathscr{K}(X)$ such that

$$
\hat{A} A_{0}=I-K, \quad \text { on } X \text {. }
$$

This leads to

$$
(\hat{A}+\hat{J}) A_{0}=I-K+\hat{J} A_{0}=I-\mathscr{Q} \text { on } X .
$$

Next, it follows from (3.2) that $\hat{A} A_{0} \in \Phi^{b}(X)$ and $i\left(\hat{A} A_{0}\right)=0$. Hence, the use of [24, Theorem 3.4, page 117] together with the Atkinson theorem [24, Theorem 2.3, page 111] implies that $A_{0} \in \Phi^{b}\left(X, X_{A}\right)$ and

$$
i(\hat{A})=-i\left(A_{0}\right) .
$$


On the other hand, since $\hat{J} \in A \mathscr{F}(X)$ and $A_{0} \in \mathscr{L}\left(X, X_{A}\right)$, applying Proposition 1.1 we get $\hat{J} A_{0} \in \mathscr{F}^{b}(X)$. Using the fact that $\mathscr{F}^{b}(X)$ is a closed two-sided ideal of $\mathscr{L}(X)$ containing $\mathscr{K}(X)$ [8] we infer that $\mathscr{Q} \in \mathscr{F}^{b}(X)$. Therefore applying [17, Proposition p:3.1 (i)] to (3.3) we get $(\hat{A}+\hat{J}) A_{0} \in \Phi^{b}(X)$ and $i\left[(\hat{A}+\hat{J}) A_{0}\right]=0$. Since $A_{0} \in \Phi^{b}\left(X, X_{A}\right)$, it follows from [24, Theorem 3.4, page 117] and the Atkinson theorem that $\hat{A}+\hat{J} \in \Phi^{b}\left(X_{A}, X\right)$ and

$$
i(\hat{A}+\hat{J})=-i\left(A_{0}\right) .
$$

Now using (3.4), (3.5) and (3.1) we find that $i(A+J)=i(A)$ which completes the proof.

PROOF OF THEOREM 2.1. The proofs of items (ii), (iii), (iv) and the first part of (i) for $i=4$ use Lemma 3.1 and are immediate. So, they are omitted.

Next, we prove (i) for $i=5$. If $\lambda \notin \sigma_{e 5}(A)$, then $\lambda \in \rho_{5}(A)$ that is $\lambda \in \Phi_{A}$ and $i(\lambda-A)=0$. Since $J \in A \mathscr{F}(X)$, applying Lemma 3.1 (i) we infer that $\lambda \in \Phi_{A+J}$ and $i(\lambda-A-J)=0$, and therefore $\lambda \notin \sigma_{e 5}(A+J)$. Thus $\sigma_{e s}(A+J) \subseteq \sigma_{e 5}(A)$. Similarly, if $\lambda \notin \sigma_{e 5}(A+J)$, then using Lemma 3.1 (i) and arguing as above we derive the opposite inclusion $\sigma_{e s}(A) \subseteq \sigma_{e s}(A+J)$.

To prove the statement for $i=6$, we first observe that the preceding step implies that $C \sigma_{e s}(A+J)=C \sigma_{e s}(A)$. This set contains points of $\rho(A)$ and $\rho(A+J)$. Accordingly, since $\alpha(\lambda-A)$ and $\beta(\lambda-A)$ (respectively $\alpha(\lambda-A-J)$ and $\beta(\lambda-A-J)$ ) are constant on any component of $\Phi_{A}$ (respectively $\Phi_{A+J}$ ) except possibly on a discrete set of points at which they have larger values (see [7, Theorem 3.3]), it cannot contain points of $\sigma_{e 6}(A)$ or $\sigma_{e 6}(A+J)$. This together with the inclusions $\sigma_{e 5}(A) \subseteq \sigma_{e 6}(A)$ and $\sigma_{e 5}(A+J) \subseteq \sigma_{e 6}(A+J)$ leads to $\sigma_{e 5}(A)=\sigma_{e 6}(A)$ and $\sigma_{e 5}(A+J)=\sigma_{e 6}(A+J)$ and the result follows.

Proof OF TheOREM 2.2. Set $\mathscr{O}:=\bigcap_{J \in \mathscr{g}(X)} \sigma(A+J)$.

Clearly, (2.1) and (2.3) show that $\mathscr{O} \subseteq \sigma_{e 5}(A)$. So, we have only to prove that $\sigma_{e s}(A) \subseteq \mathscr{O}$. If $\lambda_{0} \notin \mathscr{O}$, then there exists $J \in \mathscr{J}(X)$ such that $\lambda_{0} \in \rho(A+J)$. Let $x \in X$ and put $y=\left(\lambda_{0}-A-J\right)^{-1} x$. It follows from the estimate

$$
\begin{aligned}
\|y\|_{A+J} & =\|y\|+\|(\hat{A}+\hat{J}) y\|=\|y\|+\left\|x-\lambda_{0} y\right\| \\
& =\left\|\left(\lambda_{0}-\hat{A}-\hat{J}\right)^{-1} x\right\|+\left\|x-\lambda_{0}\left(\lambda_{0}-\hat{A}-\hat{J}\right)^{-1} x\right\| \\
& \leq\left(1+\left(1+\left|\lambda_{0}\right|\right)\left\|\left(\lambda_{0}-\hat{A}-\hat{J}\right)^{-1}\right\|\right)\|x\|
\end{aligned}
$$

that $\left(\lambda_{0}-\hat{A}-\hat{J}\right)^{-1} \in \mathscr{L}\left(X, X_{A+J}\right)$. Moreover, since $J \in \mathscr{J}(X) \subseteq A \mathscr{F}(X)$, applying Proposition 1.1 we conclude that $\left(\lambda_{0}-\hat{A}-\hat{J}\right)^{-1} \hat{J} \in \mathscr{F}^{b}\left(X_{A}, X_{A+J}\right)$. Let $\mathscr{I}$ 
denote the imbedding operator which maps every $x \in X_{A}$ onto the same element $x \in X_{A+J}$. Clearly we have $N(\mathscr{I})=\{0\}$ and $R(\mathscr{I})=X_{A+J}$. So, the estimate

$$
\begin{aligned}
\|\mathscr{I}(x)\|_{X_{A+J}} & =\|x\|_{X_{A+1}} \leq\|x\|_{X}+\|A x\|_{X}+\|J x\|_{X} \\
& \leq\left(1+\|J\|_{\mathscr{S}\left(X_{A}, X\right)}\right)\|x\|_{X_{A}}, \quad \forall x \in X_{A}
\end{aligned}
$$

leads to $\mathscr{I} \in \Phi^{b}\left(X_{A}, X_{A+\jmath}\right)$ and $i(\mathscr{I})=0$.

Next, remembering that $\left(\lambda_{0}-\hat{A}-\hat{J}\right)^{-1} \hat{J} \in \mathscr{F}^{b}\left(X_{A}, X_{A+J}\right)$ and using Lemma 3.1 (i) we get

$$
\mathscr{I}+\left(\lambda_{0}-\hat{A}-\hat{J}\right)^{-1} \hat{J} \in \Phi^{b}\left(X_{A}, X_{A+J}\right) \text { and } i\left(\mathscr{I}+\left(\lambda_{0}-\hat{A}-\hat{J}\right)^{-1} \hat{J}\right)=0
$$

On the other hand, since $\lambda_{0} \in \rho(A+J)$, it follows from (3.1) that

$$
\left(\lambda_{0}-\hat{A}-\hat{J}\right) \in \Phi^{b}\left(X_{A+J}, X\right) \text { and } i\left(\lambda_{0}-\hat{A}-\hat{J}\right)=0 .
$$

Thus, writing $\lambda_{0}-\hat{A}$ in the form

$$
\lambda_{0}-\hat{A}=\left(\lambda_{0}-\hat{A}-\hat{J}\right)\left(\mathscr{I}+\left(\lambda_{0}-\hat{A}-\hat{J}\right)^{-1} \hat{J}\right)
$$

and using (3.6), (3.7) and the Atkinson theorem we get $\lambda_{0}-\hat{A} \in \Phi^{b}\left(X_{A}, X\right)$ and $i\left(\lambda_{0}-\hat{A}\right)=0$. Now using (3.1) we infer that $\left(\lambda_{0}-A\right) \in \Phi(X)$ and $i\left(\lambda_{0}-A\right)=0$, that is, $\sigma_{e s}(A) \subseteq \mathscr{O}$.

\section{Application to singular transport equations}

The aim of this section is to apply Proposition 2.1 to study the essential spectra of the following singular neutron transport operator (see $[2,18,19]$ )

$$
A \psi(x, \xi)=-v \cdot \nabla_{x} \psi(x, v)-\sigma(v) \psi(x, v)+\int_{\mathbb{R}^{n}} \kappa\left(v, v^{\prime}\right) \psi\left(x, v^{\prime}\right) d \mu\left(v^{\prime}\right),
$$

where $(x, v) \in D \times \mathbb{R}^{n}, D$ is an open bounded subset of $\mathbb{R}^{n}, d \mu(\cdot)$ is a bounded positive Radon measure on $\mathbb{R}^{N}$ and $K$ denotes the integral part of $A$. This operator describes the transport of particles (neutrons, photons, molecules of gas, etc.) in the domain $D$. The function $\psi(x, v)$ represents the number (or probability) density of gas particles having the position $x$ and the velocity $v$. The functions $\sigma(\cdot)$ and $\kappa(\cdot, \cdot)$ are called, respectively, the collision frequency and the scattering kernel.

The main feature is that the collision frequency $\sigma(\cdot)$ and the collision operator $K$ are unbounded. Actually, an unbounded collision frequency $\sigma(\cdot)$ acts as a strong absorption which allows the unboundedness of $K$. We assume that the scattering 
kernel $\kappa(\cdot, \cdot)$ is nonnegative and there exist a closed subset $\mathscr{O} \subset \mathbb{R}^{n}$ with zero $d \mu$ measure and a constant $\sigma_{0}>0$ such that

$$
\sigma(\cdot) \in L_{\mathrm{loc}}^{\infty}\left(\mathbb{R}^{n} \backslash \mathscr{O}\right), \quad \sigma(v)>\sigma_{0} \text { a.e. }
$$

and

$$
v \rightarrow\left[\int_{\mathbb{R}^{n}}\left(\frac{\kappa\left(v, v^{\prime}\right)}{\sigma\left(v^{\prime}\right)^{1 / p}}\right)^{q} d \mu\left(v^{\prime}\right)\right]^{1 / q} \in L_{p}\left(\mathbb{R}^{n}\right)
$$

where $q$ denotes the conjugate exponent of $p$.

We denote by $K$ the following collision operator

$$
K: \psi \rightarrow K \psi(v):=\int_{\mathbb{R}^{n}} \kappa\left(v, v^{\prime}\right) \psi\left(x, v^{\prime}\right) d \mu\left(v^{\prime}\right) \in L_{p}\left(\mathbb{R}^{n}\right),
$$

where $L_{p}\left(\mathbb{R}^{n}\right):=L_{p}\left(\mathbb{R}^{n}, d \mu(v)\right)$ and we introduce the following weighted space

$$
L_{p}^{\sigma}\left(\mathbb{R}^{n}\right):=L_{p}\left(\mathbb{R}^{n}, \sigma(v) d \mu(v)\right) .
$$

It follows from the assumption (4.2) that $K \in \mathscr{L}\left(L_{p}^{\sigma}\left(\mathbb{R}^{n}\right) ; L_{p}\left(\mathbb{R}^{n}\right)\right)$ and

$$
\|K\|_{\mathscr{L}\left(L_{p}^{o}\left(\mathbb{R}^{n}\right) ; L_{p}\left(\mathbb{R}^{n}\right)\right)} \leq\left\|\left[\int_{\mathbb{R}^{n}}\left(\frac{\kappa\left(\cdot, v^{\prime}\right)}{\sigma\left(v^{\prime}\right)^{1 / p}}\right)^{q} d \mu\left(v^{\prime}\right)\right]^{1 / q}\right\|_{L_{p}\left(\mathbb{R}^{n}\right)} .
$$

We also define the space $X_{p}^{\sigma}:=L_{p}\left(D \times \mathbb{R}^{n}, \sigma(v) d x d \mu(v)\right)$. Using the boundedness of $D$ we find that $K \in \mathscr{L}\left(X_{p}^{\sigma} ; X_{p}\right)$ with

$$
\|K\|_{\mathscr{L}\left(X_{p}^{\prime} ; X_{\nu}\right)} \leq\left\|\left[\int_{\mathbb{R}^{n}}\left(\frac{\kappa\left(\cdot, v^{\prime}\right)}{\sigma\left(v^{\prime}\right)^{1 / p}}\right)^{q} d \mu\left(v^{\prime}\right)\right]^{1 / q}\right\|_{L_{p}\left(\mathbb{R}^{n}\right)},
$$

where $X_{p}:=L_{p}\left(D \times \mathbb{R}^{n} ; d x d \mu(v)\right)$.

Before going further we first recall the relevant functional setting of the problem. Let $W_{p}=\left\{\psi \in X_{p}\right.$ such that $\left.v . \nabla_{x} \psi \in X_{p}\right\}$ and define the set $\Gamma_{-}$by $\Gamma_{-}=\{(x, v) \in$ $\left.\partial D \times \mathbb{R}^{n}, v \cdot v_{x}<0\right\}$, where $v_{x}$ stands for the outer unit normal vector at $x \in \partial D$.

Next we introduce the following subspace of $W_{p}$

$$
W_{p}^{0}:=\left\{\psi \in W_{p} \text { such that } \psi_{\mid \Gamma_{-}}=0\right\} .
$$

Now we are in a position to define the streaming operator $T$

$$
\left\{\begin{array}{l}
T \psi(x, v)=-v . \nabla_{x} \psi(x, v)-\sigma(v) \psi(x, v), \quad \psi \in D(T) ; \\
D(T)=W_{p}^{0} \cap X_{p}^{\sigma} .
\end{array}\right.
$$


Note that a simple calculation using the assumption (4.1) shows that $X_{p}^{\sigma}$ is a subset of $X_{p}$ and the embedding $X_{p}^{\sigma} \hookrightarrow X_{p}$ is continuous.

Let $\lambda \in \mathbb{C}$ and consider the following boundary value problem

$$
\left\{\begin{array}{l}
\lambda \psi(x, v)+v \cdot \nabla_{x} \psi(x, v)+\sigma(v) \psi(x, v)=\varphi(x, v), \\
\psi_{i \Gamma_{-}}=0
\end{array}\right.
$$

where $\varphi$ is an assigned function in $X_{p}$, and the unknown function $\psi$ must be sought in $D(T)$.

For $\operatorname{Re} \lambda+\sigma_{0}>0,(4.3)$ can be solved formally to give

$$
\begin{aligned}
\psi(x, v)= & \psi\left(x-t^{-}(x, v) v, v\right) e^{-(\lambda+\sigma(v)) t^{-}(x, v)} \\
& +\int_{0}^{t^{-}(x, v)} e^{-(\lambda+\sigma(v)) s} \varphi(x-s v, v) d s,
\end{aligned}
$$

where $t^{-}(x, v)=\sup \{t>0, x-s v \in D, 0<s<t\}$. Since $\psi$ must belong to $D(T)$, then it follows that $\psi\left(x-t^{-}(x, v) v, v\right)=0$ for any $(x, v) \in D \times \mathbb{R}^{n}$. Accordingly, the solution of $(4.3)$ is given by

$$
\psi(x, v)=\int_{0}^{r^{-(x, v)}} e^{-(\lambda+\sigma(v)) s} \varphi(x-s v, v) d s .
$$

An immediate consequence of these facts is that $\sigma(T) \subseteq\left\{\lambda \in \mathbb{C}: \operatorname{Re} \lambda \leq-\sigma_{0}\right\}$. Since $\sigma(\cdot)$ is bounded below by $\sigma_{0}$, a similar reasoning to [11, Corollary 12.11 , page 272] shows that $\sigma(T)=\left\{\lambda \in \mathbb{C}: \operatorname{Re} \lambda \leq-\sigma_{0}\right\}$. In fact, we can easily check that $\sigma(T)$ is reduced to $\sigma C(T)$ (the continuous spectrum of $T$ ), that is, $\sigma(T)=\sigma C(T)$ (see [11, Chapter 12]). Since all essential spectra are enlargement of the continuous spectrum we infer that

$$
\sigma_{e i}(T)=\left\{\lambda \in \mathbb{C} \text { such that } \operatorname{Re} \lambda \leq-\sigma_{0}\right\}, \quad i=1, \ldots, 6 .
$$

From now on, we will assume that the measure $d \mu$ satisfies

$$
\left\{\begin{array}{l}
\text { the hyperplanes have zero } d \mu \text {-measure, that is, for each } e \in S^{n-1}, \\
d \mu\left\{v \in \mathbb{R}^{n}, \text { v.e }=0\right\}=0,
\end{array}\right.
$$

where $S^{n-1}$ denotes the unit sphere of $\mathbb{R}^{n}$. Note that condition (4.5) is not restrictive. It is, in particular, satisfied by the usual continuous, multigroup and discrete models.

Now we are in position to state the main result of this section.

THEOREM 4.1. Let $D$ be a bounded subset of $\mathbb{R}^{n}$ and $1<p<\infty$. If hypotheses (4.1), (4.2) and (4.5) are satisfied and the collision operator $K: L_{p}^{\sigma}\left(\mathbb{R}^{n}\right) \rightarrow L_{p}\left(\mathbb{R}^{n}\right)$ is compact, then,

$$
\sigma_{e i}(A)=\left\{\lambda \in \mathbb{C} \text { such that } \operatorname{Re} \lambda \leq-\sigma_{0}\right\}, \quad i=1, \ldots, 5 .
$$


To establish Theorem 4.1 the following result is required.

PROPOSITION 4.1. Assume that the hypotheses of Theorem 4.1 are satisfied. Then, for any $\lambda$ satisfying $\operatorname{Re} \lambda>-\sigma_{0}$, the operator $K(\lambda-T)^{-1}$ is compact on $X_{p}$, $1<p<\infty$.

REMark 4.1. (i) Note that the statement of Proposition 4.1 excludes the case $p=1$. This is due to the fact that our proof uses an averaging result $[19$, Theorem 3.2 (ii)], which is valid only for $1<p<\infty$. However, in the case where $K: L_{1}^{\sigma}\left(\mathbb{R}^{n}\right) \rightarrow L_{1}\left(\mathbb{R}^{n}\right)$ is positive and weakly compact, and $d \mu$ satisfies the following geometrical property

$$
\int_{\alpha_{1} \leq|x| \leq \alpha_{2}} d \mu(x) \int_{0}^{\alpha_{3}} \chi_{A}(t x) d t \rightarrow 0 \quad \text { as }|A| \rightarrow 0
$$

for every $\alpha_{1}<\alpha_{2}<\infty$ and $\alpha_{3}<\infty$, where $|A|$ is the Lebesgue measure of $A$ and $\chi_{A}(\cdot)$ denotes the characteristic function of $A, \operatorname{Lods}[18$, Corollary 2.1] proved that $K(\lambda-T)^{-1} K: X_{1}^{\sigma} \rightarrow X_{1}$ is weakly compact.

(ii) It should be noticed that Proposition 4.1 contains also information concerning the asymptotic spectrum of $A$. In particular, it tells us that

$$
\sigma(A) \cap\left\{\lambda \in \mathbb{C}: \operatorname{Re} \lambda>-\sigma_{0}\right\}
$$

consists of at most a countable set of isolated eigenvalues with finite algebraic multiplicity. This follows from [26, Theorem II].

PROOF OF PROPOSITION 4.1. Since $K$ is compact from $L_{p}^{\sigma}\left(\mathbb{R}^{n}\right)$ into $L_{p}\left(\mathbb{R}^{n}\right)$, by using linearity and approximation arguments we may restrict ourselves to the case when the scattering kernel has the form $\kappa\left(v, v^{\prime}\right)=f(v) g\left(v^{\prime}\right)$, where $f(\cdot) \in L_{p}\left(\mathbb{R}^{n}\right)$, $g(\cdot) \sigma(\cdot)^{1 / q} \in L_{q}\left(\mathbb{R}^{n}\right)$ and $q$ denotes the exponent conjugate of $p$. Again, the use of a density argument allows us to assume that $f(\cdot) \in \mathscr{C}_{c}\left(\mathbb{R}^{n}\right), g(\cdot) \sigma(\cdot)^{1 / q} \in \mathscr{C}_{c}\left(\mathbb{R}^{n}\right)$ (continuous functions with compact support). In these conditions, operator $K(\lambda-T)^{-1}$ maps $X_{\eta}$ into itself for all $\eta \in[1,+\infty]$. Using interpolation arguments (see [14, Theorem 3.10, pager 57]) we can restrict ourselves to the case $p=2$.

Let $\mathscr{A}_{g}$ be the averaging operator

$$
\mathscr{A}_{g}: \varphi \in X_{2}^{\sigma} \rightarrow \int \varphi\left(x, v^{\prime}\right) g\left(v^{\prime}\right) d \mu\left(v^{\prime}\right) \in L_{2}(D) .
$$

It suffices to show that $\mathscr{A}_{g}\left(\lambda-T_{H}\right)^{-1}$ is a compact operator from $X_{2}$ into $L_{2}(D)$. This amounts to $\mathscr{A}_{g}: D(T)=W_{2}^{0} \cap X_{2}^{\sigma} \rightarrow L_{2}(D)$ is compact.

Note that $D(T)$ equipped with the norm

$$
\|\psi\|_{D(T)}=\|\psi\|_{w_{2}^{0}}+\|\psi\|_{X_{2}^{\sigma}} \quad \forall \psi \in D(T),
$$


where $\|\psi\|_{w_{2}^{0}}=\|\psi\|_{X_{2}}+\left\|v \cdot \nabla_{x} \psi\right\|_{X_{2}}$ is a Banach space.

If $U$ is a bounded subset of $D(T)$, then there exists $\rho>0$ such that $\|\psi\|_{D(\tau)} \leq \rho$ for every $\psi \in U$. This implies, in particular, that $U$ is bounded as a set of $W_{2}^{0}$. Now applying [19, Theorem 3.2 (ii)] one sees that $\mathscr{A}_{g} U$ is relatively compact in $L_{2}(D)$. This shows the compactness of $\mathscr{A}_{g}$ which ends the proof.

ProOF OF THEOREM 4.1. Let $\lambda \in \rho(T)$. Since the collision operator $K$ is Tdefined, by the closed graph theorem we have $K(\lambda-T)^{-1} \in \mathscr{L}\left(X_{p}\right)$. On the other hand, in [18, Proposition 3.3], it is proved that $D(T)$ is continuously embedded in $X_{p}^{\sigma}$ and, for any $\lambda>0$, we have $\left\|(\lambda-T)^{-1}\right\|_{\mathcal{S}\left(X_{p}: X_{p}^{\sigma}\right)} \leq(1 / \lambda q)^{1 / q}(1 / p)^{1 / p}$. So, since $X_{p}^{\sigma}$ is continuously embedded in $X_{p}$, we infer that $\lim _{\lambda \rightarrow \infty}\left\|K(\lambda-T)^{-1}\right\|_{\mathscr{L}\left(X_{p}\right)}=0$. Therefore, there exists $\lambda \in \rho(T)$ such that $r_{\sigma}\left(K(\lambda-T)^{-1}\right)<1$. Next, using Proposition 4.1 one sees that $K(\lambda-T)^{-1} \in \mathscr{K}\left(X_{p}\right) \subset \mathscr{S}\left(X_{p}\right)$. Since $\mathscr{F}_{+}\left(X_{p}\right)=$ $\mathscr{F}_{-}\left(X_{p}\right)=\mathscr{F}\left(X_{p}\right)=\mathscr{S}\left(X_{p}\right)$ (see, for example, [16, (2.9)]), the result follows from (4.4) and Proposition 2.1.

\section{References}

[1] J. W. Calkin, 'Two-sided ideals and congruences in the ring of bounded operators in Hilbert spaces', Ann. of Math. 42 (1941), 839-873.

[2] M. Chabi and M. Mokhtar-Kharroubi, 'Singular neutron transport equations in $L_{1}$ spaces', preprint, 1995.

[3] R. Dautray and J. L. Lions, Analyse mathématique et calcul numérique: Tome 9 (Masson, Paris, 1988).

[4] M. L. Demeru and B. Montagnini, 'Complete continuity of the free gas scattering operator in neutron thermalization theory', J. Math. Anal. Appl. 12 (1965), 49-57.

[5] J. Diestel and J. J. Uhl, Vector measures, Math. Surveys 15 (Amer. Math. Soc., Providence, R.I., 1977).

[6] N. Dunford and J. T. Schwartz, Linear operators. Part I (Intersciences, New York, 1958).

[7] I. Gohberg and G. Krein, 'Fundamental theorems on deficiency numbers, root numbers and indices of linear operators', Amer. Math. Soc. Transl. Ser. 213 (1960), 185-264.

[8] I. Gohberg, A. Markus and I. A. Feldman, 'Normally solvable operators and ideals associated with them', Amer. Math. Soc. Transl. Ser. 261 (1967), 63-84.

[9] W. Greenberg, C. Van der Mee and V. Protopopescu, Boundary value problems in abstract kinetic theory (Birkhäuser, Basel, 1987).

[10] K. Gustafson and J. Weidmann, 'On the essential spectrum', J. Math. Anal. Appl. 25 (1969), $121-127$.

[11] H. G. Kaper, C. G. Lekkerkerker and J. Hejtmanek, Spectral methods in linear transport theory (Birkhäuser, Basel, 1982).

[12] T. Kato, 'Perturbation theory for nullity, deficiency and other quantities of linear operators', J. Anal. Math. 6(1958), 261-322.

[13] _- Perturbation theory for linear operators (Springer, New York, 1966). 
[14] M. A. Krasnosel'skii et al., Integral operators in space of summable functions (Noordhoff, Leyden, 1976).

[15] K. Latrach, 'Essential spectra on spaces with the Dunford-Pettis property', J. Math. Anal. Appl. 233 (1999), 607-622.

[16] K. Latrach and A. Dehici, 'Relatively strictly singular perturbations, essential spectra and application', J. Math. Anal. Appl. 252 (2000), 767-789.

[17] —, 'Fredholm, semi-Fredholm perturbations and essential spectra', J. Math. Anal. Appl, 259 (2001), 277-301.

[18] B. Lods, 'On singular neutron transport equations', preprint, 2000.

[19] M. Mokhtar-Kharroubi, Mathematical topics in neutron transport theory, new aspects, Adv. Math. Sci. Appl. 46 (World Scientific, Singapore, 1997).

[20] B. S. Nagy, 'On the stability of the index of unbounded linear transformations', Acta Math. Hungar. 3 (1952), 49-52.

[21] A. Pelczynski, 'Strictly singular and strictly cosingular operators', Bull. Acad. Polon. Sci. 13 (1965), 31-41.

[22] M. Reed and B. Simon, Methods of modern mathematical physics. IV. Analysis of operators (Academic Press, New York, 1978).

[23] M. Schechter, 'On the essential spectrum of an arbitrary operator, I', J. Math. Anal. Appl. 13 (1966), 205-215.

[24] — Principles of functional analysis (Academic Press, New York, 1971).

[25] A. Suhadolc, 'Linearized Boltzmann equation in $L_{1}$ space', J. Math. Anal. Appl. 35 (1971), 1-13.

[26] I. Vidav, 'Existence and uniqueness of nonnegative eigenfunction of the Boltzmann operator', J. Math. Anal. Appl. 22 (1968), 144-155.

[27] J. I. Vladimirskii, 'Strictly cosingular operators', Soviet Math. Dokl. 8 (1967), 739-740.

[28] J. Voigt, 'On substochastic $\mathrm{c}^{0}$-semigroups and their generators', in: Semesterbericht Funktionalanalysis, Tübingen, Wintersemester (1984/1985) pp. 1-15.

[29] R. J. Whitley, 'Strictly singular operators and their conjugates', Trans. Amer. Math. Soc. 18 (1964), 252-261.

[30] F. Wolf, 'On the invariance of the essential spectrum under a change of the boundary conditions of partial differential operators', Indag. Math. 21 (1959), 142-147.

\section{Département de Mathématiques}

Université de Corse

Quartier Grossetti, BP. 52

20250 Corte

France

e-mail: latrach@univ-corse.fr 
